\title{
Economic Evaluation of Predictive Dispatch Model in MAS-based Smart Home
}

\author{
Amin Shokri Gazafroudi ${ }^{1}$, Francisco Prieto-Castrillo ${ }^{1,3}$, Tiago Pinto ${ }^{1,2}$, Aria \\ $\mathrm{Jozi}^{2}$, and Zita Vale ${ }^{2}$ \\ ${ }^{1}$ BISITE Research Group, University of Salamanca, Salamanca, Spain \\ 2 GECAD Research Group, IPP - Polytechnic of Porto, Porto, Portugal \\ ${ }^{3}$ MediaLab, Massachusetts Institute of Technology, Cambridge, Massachusetts, USA \\ \{shokri, franciscop, tpinto\}@usal.es and $\{$ arjoz, zav\}@isep.ipp.pt
}

\begin{abstract}
This paper proposes a Predictive Dispatch System (PDS) as part of a Multi-Agent system that models the Smart Home Electricity System (MASHES). The proposed PDS consists of a Decision-Making System (DMS) and a Prediction Engine (PE). The considered Smart Home Electricity System (SHES) consists of different agents, each with different tasks in the system. A Modified Stochastic Predicted Bands (MSPB) interval optimization method is used to model the uncertainty in the Home Energy Management (HEM) problem. Moreover, the proposed method to solve HEM problem is based on the Moving Window Algorithm (MWA). The performance of the proposed Home Energy Management System (HEMS) is evaluated using a JADE implementation of the MASHES.
\end{abstract}

Keywords: Home energy management system, multi-agent system, prediction engine, interval optimization, decision-making under uncertainty.

\section{Nomenclature \\ Indices \\ $t$ Index of time periods. \\ $i$ Index of Distributed Energy Resources (DERs). \\ $j$ Index of electrical loads. \\ $k$ Index of energy storage systems.}

\section{Variables}

$O F /$ Objective function.

$P_{i_{t}}$ Total power generation for DER $i$ in period $t$.

$P_{n e t_{t}}$ Power generation that is bought from local electricity market in period $t$.

$P_{k_{t}}$ Total power generation for energy storage system $k$ in period $t$.

$C_{t}^{k}$ State of charge for energy storage system $k$ in period $t$.

$L_{j_{t}}$ Electrical load $j$ in period $t$. 
$L_{j_{t}}^{\text {shed }}$ Load shedding for load $j$ in period $t$.

$S_{i_{t}}$ Spillage amount for DER $i$ in period $t$.

$D_{i_{t}}$ Difference between the scheduled day-ahead and predicted power generation for DER $i$ in time period $t$.

\section{Parameters}

$\lambda_{i_{t}} / \lambda_{\text {net }_{t}}$ Electricity price for DER $i /$ network in period $t$.

$S_{\max }$ Maximum power capacity for the line.

$P_{i_{t}}^{\text {pred }}$ Predicted power generation for DER $i$ in period $t$.

$\alpha_{i}$ Optimistic coefficient related to the power generation for DER $i$.

$\sigma_{i}$ Prediction variance related to the power generation for DER $i$.

$U_{j}^{\max }$ Maximum energy consumption for load $j$.

\section{Introduction}

In the recent decade, new visions and approaches have been raised in order to deal with new challenges due to increment of renewable energy sources. One of the most consensual solutions is the so-called Smart Grid (SG) [1]. In this scope, buildings can purchase and sell the generated energy locally [2]. Hence, they are known as prosumers- i.e. both consumers and producers. Hence, HEMS is necessary for achieving an economic improvement through automation technologies. In this sense, smart homes can control, monitor and manage the system through network communications [3], [4].

Various researches have been presented for optimal scheduling of smart home energy, and different algorithms and methods have been represented based on their goals, strategies, utilized technologies, and software. In [5], the authors have discussed about the necessities of using computational intelligence in the HEMSs. In [6], each smart home has been considered as an autonomous agent that can buy, sell, and store electricity. Furthermore, uncertainty is modeled through generating the random data and functions in [6]. In [7], HEM has been defined as an intelligent Multi-Agent System (MAS). Also, JADE [8] is used to implement the proposed model of [7]. In [9], a MAS has been used in the distribution network scale, while agents consist of home agents and retailer agents. In [9], the purpose of the authors was to minimize the payment cost of electricity. In [10], authors proposed a method to apply the local energy resources optimally

through minimizing the loss of energy. The main purpose of [10] is to minimize the purchasing cost of electricity. In [11], HEM problem in connection with transactive energy nodes has been discussed. Authors defined that transactive energy nodes are ones who have two-way communication with market and ca manage energy nodally. Moreover, co-simulation of smart homes and transactive energy market has been studied in [11].

In this paper, MASHES is defined as a class of organization-based multiagent system where each agent has different tasks in the system. It will deal with the PDS to manage electrical energy as well as the smart home ability 
to trade electrical energy in the power grid. In addition, information provider is defined as an agent which provides all required information. Hence, JADE is used to implement the proposed organization-based MASHES. Furthermore, the modified stochastic predicted bands method that has been introduced in [12] is used in conjunction with the moving window algorithm to reschedule energy in each time-window.

The rest of this paper is organized as follows. Section 2 introduces the proposed predictive dispatch model of the MASHES. Then, the MAS structure is described in Section 3. Section 4 expresses the simulation results. Finally, Section 5 provides the conclusions.

\section{Proposed Predictive Dispatch Model}

PDS is defined as an organization of agents who is in charge of managing electrical energy in the MASHES. In this model, PDS consists of two agents: PE and DMS. The predictive dispatch model is based on the moving window algorithm. According to this approach, the scheduled energy of all agents is updated in each time period. In the following, the tasks of these agents is discussed.

\subsection{Prediction Engine (PE)}

PE must provide the accurate prediction of all the stochastic variables of the system such as wind speed, solar radiation, weather temperature and electrical non-shiftable loads for DMS. Hence, the outputs of this agent will be the inputs of DMS. As distributed energy resources are non-dispatchable resources, the forecasting of its power output is very important for the DMS. Hence, accurate forecasting of PE can assist DMS to make optimum decisions. To this end, prediction methodology is used to achieve the required forecasted values (e.g., by using the Support Vector Machines [13]).

The Support Vector Machines (SVM) algorithm is implemented by a generalization of the nonlinear algorithm Generalized Portrait created by Vapnik and Lerner in the sequence of [14]. However, the SVM approach in the current form was first introduced at the COLT conference, in 1992 [15]. The information to use in an SVM must follow the format suggested in (1):

$$
\left(y_{1}, x_{1}\right), \ldots,\left(y_{i}, x_{i}\right), x_{i} \in R^{n}, y_{i} \in R
$$

Where $\mathrm{n}$ is the size of training data. For classification: $y_{i}$ assumes finite values; in binary classifications: $y_{i} \epsilon\{+1,-1\}$; in digit recognition: $y_{i} \epsilon\{1,2,3,4,5,6,7,8,9,0$ \} ; and for regression purposes, $y_{i}$ is in general a real number $y_{i} \in R$. The implementation of SVM requires considering some important aspects, namely: Feature Space, Loss Functions, and Kernel Functions. The most applicable kernels for time series forecasting, as in the problem considered in this work, are the Radial Basis Function (RBF) and the exponential Radial Basis Function (eRBF). These two kernels are specifically tailored to regression of time series data. 


\subsection{Decision-Maker System (DMS)}

The task of DMS is to make optimum decisions in the MASHES. In this case, DMS faces a discrete optimization problem. DMS reschedule the optimum decisions in each time-window based on the re-prediction outputs of the PE. Fig. 1 shows the scheduling time framework of the proposed HEM problem in the MASHES. As it is shown in Fig. 1, the time step and the interval scheduling are considered to be equal to 1 hour, while the scheduling window equals 24 hours. The proposed HEM problem includes two stages. These stages consist of Day-Ahead Scheduling (DAS) and Real-Time rescheduling Interval (RTRI) stages. DAS stage obtains the optimum decisions for the system in day d-1 without considering uncertainties of decision-making variables. However, uncertainty is considered in the RTRI stage. This way, RTRI stage is rescheduled in each period to update the optimum decisions of DMS.

Day-Ahead Scheduling Stage has an objective function that is represented in $(2)$ :

$$
O F^{s}=\sum_{t=1}^{N_{t}}\left(\sum_{i \in D E R s}\left(\lambda_{i} P_{i, \text { out }_{t}}^{s}\right)-\lambda_{\text {net }} P_{\text {net }_{t}}^{s}\right)
$$

In DAS stage, the objective function includes two parts. The first part represents the revenue of selling electrical energy produced by distributed energy resources to the electricity market. The second term expresses the costs due to buying electrical energy from/local energy market. In this model, the cost of reactive power as a major element of ancillary service has not been considered. Interested readers are referred to [16].The constraints of the DAS stage:

$$
\begin{aligned}
& P_{n e t_{t}}^{s}+\sum_{i \in D E R s} P_{i, i n_{t}}^{s}=\sum_{j \in E L s} L_{j_{t}}^{s} \\
& -S_{\text {max }} \leq P_{\text {net }_{t}}^{s}-\sum_{i \in D E R s} P_{i, \text { out }_{t}}^{s} \leq S_{\max } \\
& P_{i_{t}}^{s}=P_{i, \text { in }_{t}}^{s}+P_{i, \text { out }} \text { t }_{t} \\
& P_{i_{t}}^{\min } \leq P_{i_{t}}^{s} \leq P_{i_{t}}^{\max } \\
& L_{j_{t}}^{\min } \leq L_{j_{t}}^{s} \leq L_{j_{t}}^{\max }
\end{aligned}
$$

(3) establishes the power balance equation due to distributed energy resources, grid power input and electrical loads. Loss of power is not considered in this problem for simplicity. (4) represents the power flow limitation through the distribution line. Besides, the total power generation of each agent of the distributed energy resources is represented in (5). (6) expresses power output limitations of energy resources. Besides, (7) represents the electrical power consumed of electrical loads. 


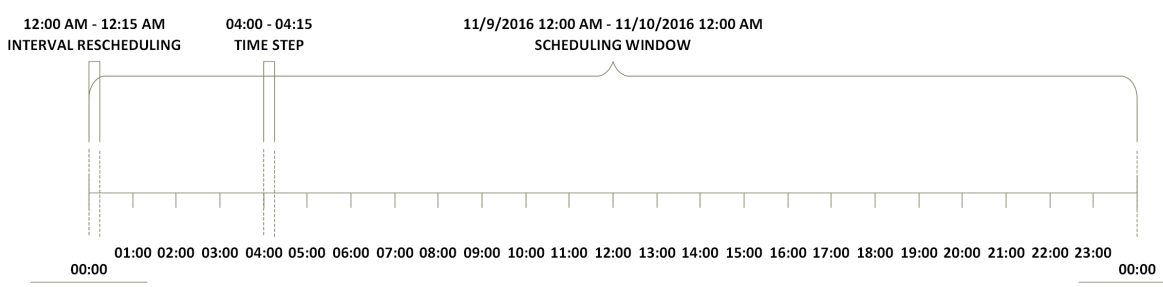

Fig. 1. Scheduling time frame.

Real-time Rescheduling Interval Stage is the second stage of HEM problem. In this stage, the decision-making variables are determined from the outputs of the first stage and the uncertainty in the Real-Time (RT) operation. Moreover, optimum decisions are rescheduled in each period in this stage. The objective function of the RTRI stage, $O F^{r t}$, is represented as follows:

$$
\begin{aligned}
& O F^{r t}=\sum_{t=1}^{N_{t}} o f_{t}=\sum_{i \in D E R s} \lambda_{i} P_{i, \text { out }}-\lambda_{\text {net }} P_{\text {net }} \\
& +\sum_{k \in E S S s} \lambda_{k} P_{k, \text { out }_{t}}-\sum_{j \in E L s} V O L L_{j} L_{j_{t}}^{\text {shed }}-\sum_{i \in D E R s} V_{i}^{s} S_{i_{t}} \\
& O F_{h}^{r t}=\sum_{t=h}^{N_{t}} o f_{t}
\end{aligned}
$$

(8) expresses objective function in each time-period. $O F^{r t}$ consists of five parts. The first part represents the revenue of selling energy produced by distributed energy resources to the market. The total cost of electrical energy that is bought from market is presented in the second part. The third part represents the profit due to selling the stored electrical energy to the market. The Value Of Loss Load (VOLL) is stated in the fourth part. Finally, spillage cost, $V_{i}^{s}$, of energy resources are introduced in the last part. The objective function of the RTRI stage is updated in each period. Hence, $O F_{h}^{r t}$ is the updated objective function of the RTRI stage in $h^{\text {th }}$ time period. Also, the power balance equation in the RTRI stage is expressed in (10). The power flow limitation through distribution line in the RT is expressed in (11).

$$
\begin{aligned}
& P_{n e t_{t}}^{r t}+\sum_{i \in D E R s} P_{i, i n_{t}}^{r t}+\sum_{k \in E S S s} P_{k, \text { in }_{t}}^{r t}=\sum_{j \in E L s}\left(L_{j_{t}}^{r t}-L_{j_{t}}^{\text {shed }}\right) \\
& -S_{\text {max }} \leq P_{\text {net }}^{r t}-\sum_{i \in D E R s} P_{i, \text { out }_{t}}^{r t}-\sum_{k \in E S S s} P_{k, \text { out }}^{r t} \leq S_{\text {max }}
\end{aligned}
$$

DERs generate electricity power in the smart home. The power output of DERs in the RTRI stage, $P_{i_{t}}^{r t}$, is obtained based on (12). From (12), $P_{i_{t}}^{r t}$ is the 
power output of DER in the RTRI, and $S_{i_{t}}$ is the spillage power of the DER. (13) represents the maximum and minimum power limitations of the DER. (14) represents that total power output of the DER equals its power output consumed in the home and the amount of power generation sold. The spillage amount of DER is the amount of power generation that is generated by DER but it is not used because of technical and economic constraints in period $t$ as presented in (15).

$$
\begin{aligned}
& P_{i_{t}}^{r t}=P_{i, p_{t}}^{r t}-S_{i_{t}} \\
& P_{i_{t}}^{\text {min }} \leq P_{i, p_{t}}^{r t} \leq P_{i_{t}}^{\text {max }} \\
& P_{i_{t}}^{r t}=P_{i, i_{t}}^{r t}+P_{i, \text { out }}^{r t} \\
& 0 \leq S_{i_{t}} \leq P_{i, p_{t}}^{r t}
\end{aligned}
$$

In this model, the MSPB method is used to model the uncertainty of variables in the HEM problem. As highlighted, the proposed interval method to model uncertainty of DERs has been defined for the first time in [12]. This is why the performance of MSPB is explained briefly in this section. Hence, in this approach, the uncertainty of stochastic variables is modeled based on their predicted amounts. Therefore, the prediction amounts of DERs' power generation that come from the $\mathrm{PE}$ are used in the formulations of MSPB. Also, $\sigma_{i}^{u p}$ and $\sigma_{i}^{\text {down }}$ parameters state the amounts of upper and lower variances of the predicted variables with respect to their actual amounts, respectively. Then, the difference between the day-ahead DERs' power generation, $P_{i_{t}}^{d a}$, and their predicted amount for each time, $P_{i_{t}}^{\text {pred }}$, is determined as (16).

$$
D_{i_{t}}=P_{i_{t}}^{s}-P_{i_{t}}^{\text {pred }}
$$

Moreover, $\alpha_{i}$ is defined as a slack variable for DMS to handle the stochastic behavior of the DER power generation. Therefore, $\alpha_{i}$ is labeled an optimistic coefficient with values between 0 and 1 . In this paper, outdoor temperature and must-run services are considered as deterministic variables for simplicity. However, uncertainty of DERs is considered based on (17).

$$
\begin{cases}P_{i_{t}}^{\text {pred }} \alpha_{i}+\left(P_{i_{t}}^{\text {pred }}-\sigma_{i}^{\text {up }}\right)\left(1-\alpha_{i}\right) \leq P_{i_{t}}^{b} & \\ \leq\left(P_{i_{t}}^{\text {pred }}+\sigma_{i}^{\text {down }}\right) \alpha_{i}+P_{i_{t}}^{\text {pred }}\left(1-\alpha_{i}\right) & D_{i_{t}} \geq 0 \\ \left(P_{i_{t}}^{\text {rred }}-\sigma_{i}^{\text {up }}\right) \alpha_{i}+P_{i_{t}}^{\text {pred }}\left(1-\alpha_{i}\right) \leq P_{i_{t}}^{b} & \\ \leq P_{i_{t}}^{\text {pred }} \alpha_{i}+\left(P_{i_{t}}^{\text {pred }}+\sigma_{i}^{\text {down }}\right)\left(1-\alpha_{i}\right) & D_{i_{t}} \leq 0\end{cases}
$$

Energy Storage Systems (ESSs) can be utilized economically based on the charge and discharge strategies in the HEM problem. The power generation of ESSs is expressed in (18) and (25). (19) represents the state of charge balance equation in a ESS, and $C_{i}^{k}$ is an initial state of charge in the ESS. Maximum and minimum limitations of discharge current of the ESS are represented in (20) and (22). Also, (21) and (23) express constraints of ESS in the charge state. 


$$
\begin{aligned}
& P_{k_{t}}^{r t}=-P_{k, b_{t}}^{r t}-\omega_{t}^{c}+\omega_{t}^{d}+\omega_{t}^{m} \\
& C_{t}^{k}=C_{t-1}^{k}+\omega_{t}^{c} \eta_{G 2 V}-\omega_{t}^{d} / \eta_{V 2 G}-\omega_{t}^{m} / \eta_{V 2 T}, t \geq 2 \\
& C_{t}^{k}=C_{i}^{k}, t=1 \\
& P_{k, d_{t}}^{\min } \eta_{k}\left(1-u_{t}^{k}\right) \leq \omega_{t}^{d} \leq P_{k, d_{t}}^{\max } \eta_{k}\left(1-u_{t}^{k}\right) \\
& P_{k, c_{t}}^{\text {min }} \eta_{k} u_{t}^{k} \leq \omega_{t}^{c} \leq P_{k, c_{t}}^{\max } \eta_{k} u_{t}^{k} \\
& 0 \leq \omega_{t}^{d} \leq\left(C_{t}^{k}-P_{k, d_{t}}^{\min }\right) \eta_{k} \\
& 0 \leq \omega_{t}^{c} \leq\left(P_{k, c_{t}}^{\max }-C_{t}^{k}\right) \eta_{k} \\
& P_{k, d_{t}}^{\max }-C_{t-1}^{k} \leq P_{e v, b_{t}}^{r t} \leq P_{k, c_{t}}^{\max }-C_{t-1}^{k}, t \geq 2 \\
& P_{k_{t}}^{r t}=P_{k, \text { in }}^{r t}+P_{k, \text { out }}^{r t}
\end{aligned}
$$

Electrical Loads (ELs) include electrical consumers that can be controllable and/or shiftable. (26) and (27) represent ELs' power and energy consumed maximum and minimum limitations. Moreover, (28) expresses the load shedding constraint of ELs. (29) and (30) express equal and unequal constraints of ELs.

$$
\begin{aligned}
& L_{j_{t}}^{\text {min }} \leq L_{j_{t}}^{r t} \leq L_{j_{t}}^{\max } \\
& U_{j_{t}}^{\text {min }} \leq \sum_{t=1}^{N_{t}} L_{j_{t}}^{r t} \leq U_{j_{t}}^{\text {max }} \\
& 0 \leq L_{j_{t}}^{\text {shed }} \leq L_{j_{t}}^{r t} \\
& f_{a}\left(M_{t}^{r t}\right)=0 ; a=1, \ldots, N_{a} . M \in\left\{L_{j}, \theta_{\text {out }}, \theta_{\text {in }}\right\} . \\
& g_{b}\left(M_{t}^{r t}\right) \leq 0 ; b=1, \ldots, N_{b} .
\end{aligned}
$$

\section{Implementation of MASHES}

The MAS for HEMS allows to model different devices in a house through autonomous agents. This MAS is implemented in JADE. The architecture of the agent system is depicted in Fig. 2. The organization-based MAS is composed

by Local Electricity Market (LEM), PDS, DERs, ESSs and ELs. LEM is a set of external agents that consists in the retailer-the energy supplier- and the Demand Response (DR) aggregator. PDS is a group of agents that is in charge of connecting all the agents in a house. In addition, it analyses and predicts data. Also, the energy management is done by the PDS. DERs is responsible for renewable energy resources, such as wind and PV panels. ESSs is a set of agents, that represent the energy storage units, e.g. battery, and Electrical Vehicles $(\mathrm{EVs}) . \mathrm{ELs}$ is a group of different agents that only consume the electrical energy but whose type is different. This proposed organization-based MAS structure is 


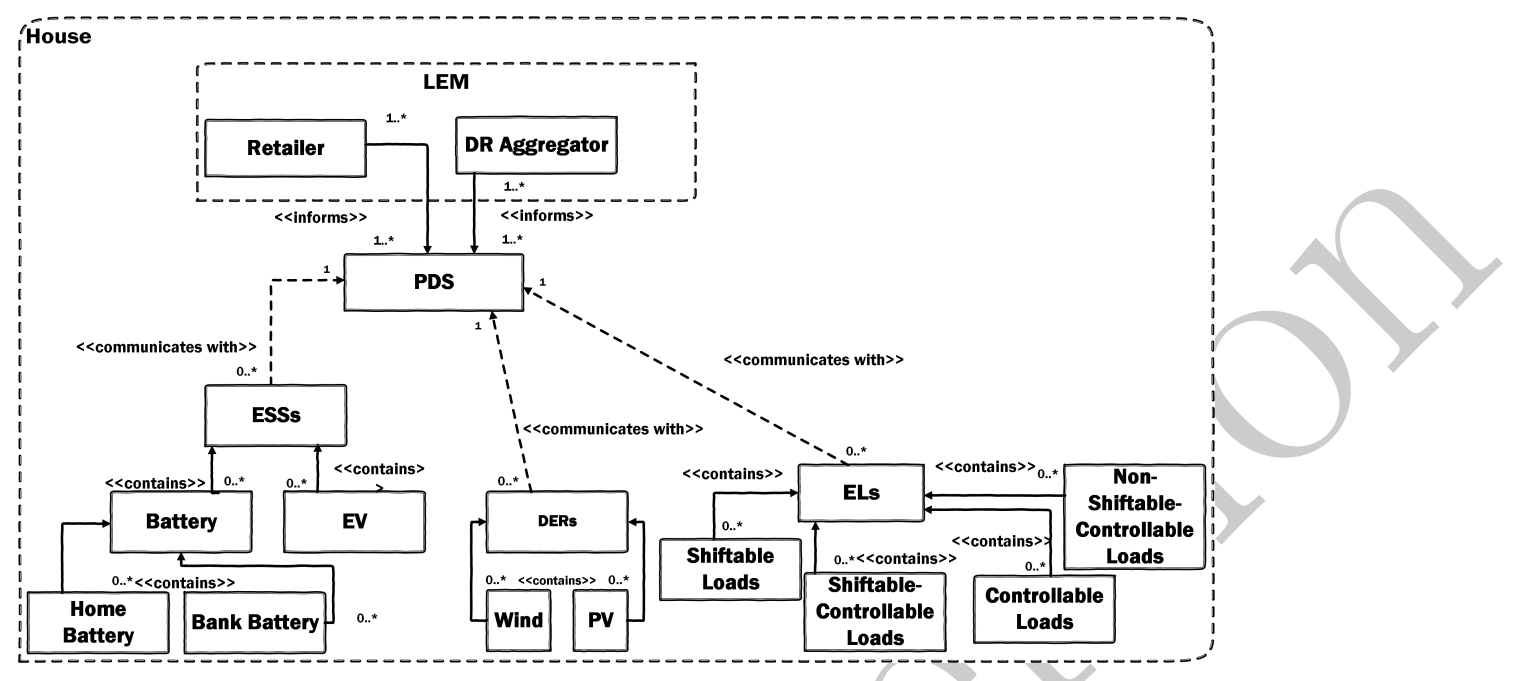

Fig. 2. MAS architecture.

also capable of interacting with the Multi-Agent Smart Grid Simulation Platform (MASGriP) [17], and the Multi-Agent Simulator of Competitive Electricity Markets (MASCEM) [18].

\section{Simulation Results}

A physical system from [19] is used to evaluate the performance of the proposed HEMS. However, some modifications of the system parameters are made. For instance, the predicted data of PV power generation and the must-run services are used from [20]. The maximum energy produced by the PV system is 2-kWh. The battery can store between 0.48 and $2.4 \mathrm{kWh}$, and its maximum charging/ discharging rates are $400 \mathrm{~W}$. Besides, charging and discharging efficiencies are $90 \%$. Maximum heating power equals $2 \mathrm{~kW}$ to maintain the temperature of the house within \pm 1 of desired temperature $\left(23^{\circ} \mathrm{C}\right)$. The thermal resistance of the building shell is equal to $18^{\circ} \mathrm{C} / \mathrm{kW}$, and $\mathrm{C}$ equals $0.525 \mathrm{kWh} /{ }^{\circ} \mathrm{C}$. The energy capacity of the storage water heater is $10.46 \mathrm{kWh}(180 \mathrm{~L})$ which has $2 \mathrm{~kW}$ heating element. The rated power of the pool pump is $1.1 \mathrm{~kW}$, and it can run

for a maximum of 6 hours during the day. The performance of the proposed HEM model is assessed in three cases. The program implemented is solved in GAMS 23.7 [21]. In this section, the performance of proposed model predictive dispatch is addressed in three cases.

Case 1: Effect of distributed energy resources and energy storage systems, Case 2: Effect of Demand Response Program (DRP), and Case 3: Effect of the connection state on the objective function of the system are evaluated.

The impact of a battery system on the objective functions is shown in Fig. $3(\mathrm{a})$. The battery system increases the amount of the objective function. How- 

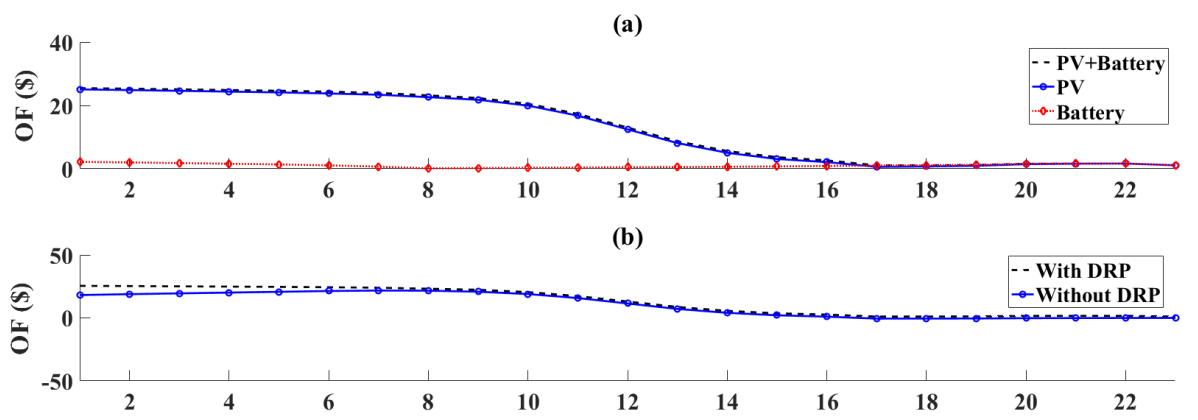

(c)

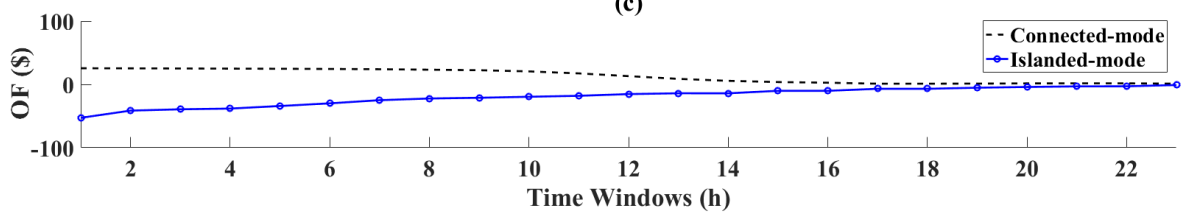

Fig. 3. Impact of (a) DERs and ESSs, (b) DRP and (c) connection state on Objective Function (OF) in HEM problems.

ever, the impact of PV system on the objective function is more evident. Fig. 3(b) expresses that considering DRP in the HEMS causes to increase the amount of objective function. The impact of DRP is more obvious after $17^{\text {th }}$ time-windows that the objective function is negative without considering DRP in the problem, while the objective function is alway positive in case (a) even after $17^{\text {th }}$ time-windows that the power generation of PV system equals zero. Additionally, Fig. 3(c) displays the influence of the connection state of the smart home to the power grid. As shown in Fig. 3(c), the objective function is negative in all time-windows because it is impossible for MASHES to sell electricity to the market. Hence, the effects of battery system is less obvious in case (c). Also, MASHES is faced spillage cost due to surplussing electricity generation of PV system, and load shedding cost.

\section{Conclusions}

The performance of the proposed HEM model has been evaluated based on the impacts of DERs, ESSs, DRP, and the connection state of the smart home to the power grid. From the simulation, considering DRP and the battery system increases the amount of the objective function. However, the influence of DRP in this case is more evident. Besides, the objective function is negative in all time-windows when the smart home is in islanded- mode. In our future work, the control unit will be added to the predictive dispatch system to control noises due to inaccuracy of prediction outputs in real-time operation. 


\section{Acknowledgment}

This work has been supported by the European Commission H2020 MSCARISE-2014: Marie Sklodowska-Curie project DREAM-GO Enabling Demand Response for short and real-time Efficient And Market Based Smart Grid Operation - An intelligent and real-time simulation approach ref. 641794. Moreover, the authors would like to thank Dr. Juan Manuel Corchado of University of Salamanca for his thoughtful suggestions and supports.

\section{References}

1. S. Borlase (editor), "Smart Grids Infrastructure, Technology, and Solutions," CRC Press, 2013.

2. K. Kok, S. Karnouskos, D. Nestle, A. Dimeas, A. Weidlich, C. Warmer, P. Strauss, B. Buchholz, S. Drenkard, N. Hatziargyriou, V. Lioliou, "Smart houses for a smartgrid," 20th International Conference on Electricity Distribution, CIRED 2009.

3. S. K. Das, D. J. Cook, A. Battacharya, E. O. Heierman, "The role of predictionalgorithms in the MavHome smart home architecture," IEEE Wireless Communications, vol. 9, n. 6, pp. 7784, 2002.

4. W. Young-Min, L. Jong-Uk, J. Sung-Kwan, "Electric vehicle charging method for smart homes/buildings with a photovoltaic system," IEEE Transactions on Consumer Electronics, vol. 59, n. 2, pp. 323328, 2013.

5. M. Manic, D. Wijayasekara, K. Amarasinghe, and J. J. Rodriguez-Andina, "Building Energy Management Systems The Age of Intelligent and Adaptive Buildings," IEEE Indus. Elec. Magazine, vol. 10, no. 1, pp. 25-39, Mar. 2016.

6. S. Kahrobaee, Student Member, IEEE, R. A. Rajabzadeh, L. Soh, and S. Asgarpoor, "A Multiagent Modeling and Investigation of Smart Homes With Power Generation, Storage, and Trading Features," IEEE Trans. on Smart Grid, vol. 4, no. 2, pp. 659 - 668, June 2013.

7. W. Li, T. Logenthiran, and W. L. Woo, "Intelligent multi-agent system for smart home energy management," Inn. Smart Grid Tech.- Asia (ISGT ASIA), 3-6, Nov. 2015.

8. Java agent development framework (jade) at http://jade.tilab.com/

9. Zh. Wang, and R. Paranjape, "Optimal Residential Demand Response for Multiple Heterogeneous Homes With Real-Time Price Prediction in a Multiagent Framework," IEEE Trans. on Smart Grid, vol. PP, no. PP, pp. 1-12, Oct. 2015.

10. Ch. Zhao, Sh. Dong, F. Li, and Y. Song, "Optimal Home Energy Management System with Mixed Types of Loads," CSEE Journal of Power and Energy Sys., vol. 1, no. 4, pp. 1-11, Dec. 2015.

11. A. Pratt, D. Krishnamurthy, M. Ruth, H. Wu, Monte Lunacek, and P. Vaynshenk, "Transactive Home Energy Management Systems," IEEE Elec. Magazine, vol. 4, no. 4, pp. 8-14, Dec. 2016.

12. Amin Shokri Gazafroudi, F. Prieto-Castrillo, and J. M. Corchado, "Residential Energy Management Using a Novel Interval Optimization Method," Accepted: 4th International Conference on Control, Decision and Inf. Tech., 2017 (CoDIT'17).

13. Tiago Pinto, Tiago M. Sousa, Isabel Praa, Zita Vale, Hugo Morais, "Support Vector Machines for Decision Support in Electricity Markets' Strategic Bidding," Neurocomputing, vol. 172, pp. 438-445, January 2016. 
14. Vapnik, V., A. Lerner, A., "Pattern recognition using generalized portrait method. Automation and Remote Control," 24, 774780, 1963.

15. Boser, B.E., Guyon, I.M., Vapnik, V.N., "A Training algorithm for optimal margin classifiers," COLT conference, 1992.

16. B. Mozafari, A.M. Ranjbar, T. Amraee, M. Mirjafari, A. R. Shirani, "A hybrid of particle swarm and ant colony optimization algorithms for reactive power market simulation," Journal of Intelligent \& Fuzzy Systems, vol. 17, no. 6, pp. 557-574, Jan. 2006.

17. P. Oliveira, T. Pinto, H. Morais, and Z. Vale, "MASGriP - A Multi-Agent Smart Grid Simulation Plataform," in IEEE Power and Energy Society General Meeting, San Diego, USA, 2012.

18. G. Santos, T. Pinto, I. Praa, and Z. Vale, "MASCEM: Optimizing the performance of a multi-agent system," Energy, vol. 111, pp. 513-524, Sep. 2016.

19. M. Pedrasa, T. Spooner, and I. MacGill, "Improved energy services provision through the intelligent control of distributed energy resources," in Proc. 2009, IEEE Bucharest power Tech conf.

20. Amin Shokri Gazafroudi, T. Pinto, F. Prieto-Castrillo, J. Prieto, J. M. Corchado, A. Jozi, Z. Vale, G. K. Venayagamoorthy, Organization-based Multi-Agent Structure of the Smart Home Electricity System, Accepted: 4th IEEE Cong. on Evolut. Comp., 2017.

21. GAMS Release 2.50. A users guide. GAMS Development Corporation,1999. Available: http://www.gams.com. 